\title{
Improvement training in Immunotherapy for healthcare professionals. Does training make a difference?
}

Ana de Carvalho Vaz ${ }^{1}$, Francisco Ribeiro-Mourão ${ }^{2}$, Ariana Barreira Teles ${ }^{3}$, Beatriz Sousa ${ }^{1}$, Ana Rita Araújo ${ }^{2}$, and Helena Ramalho ${ }^{1}$

${ }^{1}$ Unidade Local de Saude do Alto Minho EPE

${ }^{2}$ Centro Hospitalar Universitário do Porto EPE Centro Materno-Infantil do Norte Dr Albino Aroso

${ }^{3}$ Instituto Portugues de Oncologia do Porto Francisco Gentil EPE

February 23, 2022

\section{Hosted file}

Letter Improvement training in Immunotherapy for healthcare professionals.docx available at https://authorea. com/users/462011/articles/557533-improvement-training-in-immunotherapyfor-healthcare-professionals-does-training-make-a-difference 